\title{
CAUSES OF MORTALITY IN SCHIZOPHRENIA: AN UPDATED REVIEW OF EUROPEAN STUDIES
}

\author{
Patryk Piotrowski ${ }^{1}$, Tomasz M. Gondek ${ }^{1}$, Anna Królicka-Deręgowska ${ }^{1}$, Błażej Misiak ${ }^{2}$, \\ Tomasz Adamowski ${ }^{3}$ \& Andrzej Kiejna ${ }^{4}$ \\ ${ }^{1}$ Department of Psychiatry, Wroclaw Medical University, Wroclaw, Poland \\ ${ }^{2}$ Department of Genetics, Wroclaw Medical University, Wroclaw, Poland \\ ${ }^{3}$ Department of Nervous System Diseases, Faculty of Health Science, Wroclaw Medical University, Wroclaw, Poland \\ ${ }^{4}$ Institute of Psychology, University of Lower Silesia, Wroclaw, Poland
}

received: 14.12.2016;

revised: 28.12.2016;

accepted: 9.1.2017

\section{SUMMARY}

Background: The excess mortality in schizophrenia is still a phenomenon insufficiently studied on the cross-national level. It is important to analyse current studies on morality in schizophrenia since significant changes have recently taken place in psychiatric health care systems and guidelines of pharmacological treatment have been developed in European countries.

Subjects and methods: This article reviews studies addressing mortality in schizophrenia in Europe that were published in English in the Pubmed database in 2009-2014. It aimed at determining countries where studies were conducted, methodologies and tools used, and current main mortality rates, as well as direction of causality in this group of patients.

Results: The recently published studies were conducted only in few European countries. The majority of data was obtained from general medical records and death records. The studies indicate that schizophrenia patients are characterized by higher mortality rate than the general population, with natural causes (cardiovascular diseases and cancers) and suicides predominating. The increasing mortality gap with significantly shorter life expectancy of patients with schizophrenia in comparison with the general population is considerable.

Conclusions: Death records are a crucial tool in studies on mortality in schizophrenia patients; however they are insufficiently employed. Recent European reports do not show positive tendencies, indicating that standardized mortality rates in schizophrenia remain on the same level or even increase, particularly for deaths resulting from natural causes. Due to various methodologies used in studies, their direct comparison is difficult. This limitation warrants further discussion on methods used in future studies on schizophrenia mortality in Europe.

Key words: schizophrenia - mortality - life expectancy - epidemiology

\section{INTRODUCTION}

Schizophrenia is a severe mental disorder that is associated with higher suicide risk and increased prevalence of comorbid physical health disorders (Prince et al. 2007). Therefore, an average life expectancy in this population is significantly shorter in comparison with the general population. Although patient's death always represents an objective endpoint, yet mortality studies, apart from their statistical value, are used to monitor the quality of psychiatric and somatic healthcare. As recently significant changes have taken place in the psychiatric health care, including standardisation of therapies in European countries, regular analyses of mortality in schizophrenia are considered necessary. The importance of what has been started by Sykes (1840) and Farr (1841) in the $19^{\text {th }}$ century is now well acknowledged by the authors of the EuroPoPP-MH report, who, citing Korkeila et al. (2003), describe mortality as one of the basic metal health indicators which allow to monitor the efficacy of mental health policy (Samele et al. 2013). OECD also lists mortality in persons with mental disorder as one of the health care quality indicators (Samele et al. 2013). Similarly, WHO
Mental Health Action Plan 2013-2020 (WHO 2013) considers gathering mortality rates data, among others, as a crucial information for mental health systems (WHO 2013). Considering that, there is a striking lack of cross-national research in this field, apart from large studies conducted by the pharmacological companies, in which mortality is usually not the main factor measured.

The last comprehensive systematic reviews of studies on mortality in schizophrenia were published in 2007 (Saha et al. 2007) and 2010 (Bushe et al. 2010), while the only systematic review of this issue in Europe was published in 2010 (De Hert 2010), and only comprised studies concerning the effect of antipsychotic treatment on mortality. Therefore, we aimed at reviewing current European studies addressing mortality rates in schizophrenia patients that were published in the years 2009-2014 in order to answer the following questions:

1. What were the main factors associated with mortality in schizophrenia?

2. In which countries and with what methods are these studies conducted most often? 


\section{SUBJECTS AND METHODS}

Two researchers (A.K-D. and T.M.G.) independently browsed the PubMed database using the Medical Subject Headings (MeSH) tool and compared the results obtained. The articles were accepted for the systematic review if both of the researchers deemed the paper relevant. The following headings were used: Schizophrenia, with subheading: Mortality; and Europe: ("Schizophrenia/ mortality" (Mesh)) AND "Europe" (Mesh); selecting filters: abstract and full-text available, in English, concerning humans, published between January 1, 2009 and December 31, 2014. Out of 48 results returned, 26 studies were considered eligible (Figure 1). We decided to accept only papers that presented data for schizophrenia diagnosed according to ICD or DSM classifications criteria, or diagnosed clinically by a physician if no information about classification used was available. We included articles presenting data for cases of dual diagnosis, as well as those of other comorbidities, providing that the diagnosis of schizophrenia was set in line with the conditions specified above. Papers that only included data for the diagnosis of schizophrenia set on the basis of questionnaires or lacked clear indication of a clinical diagnosis were rejected. We also rejected studies which presented data for mixed samples that included

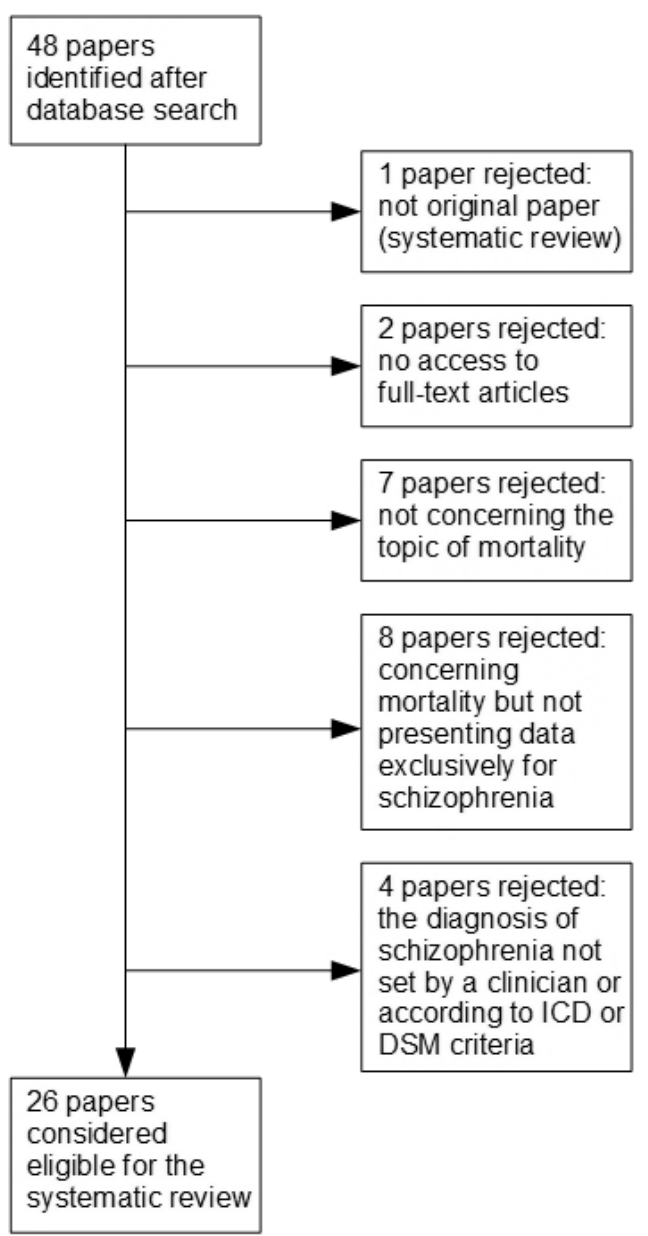

Figure 1. The process for identification of the papers eligible for the review persons with various diagnoses, one of which was schizophrenia but no exclusive mortality data for schizophrenia was available. We eventually rejected 22 papers because they either did not consider mortality directly, schizophrenia was considered jointly with other mental disorders with no exclusive mortality data or the diagnosis of schizophrenia was uncertain. Papers that only presented mortality data for other diagnoses, such as schizoaffective disorder or bipolar disorder, were also rejected. However we agreed not to reject studies regarding patients with first episode of psychosis if a diagnosis of schizophenia, according to the criteria specified above, was confirmed later during the observation period.

\section{RESULTS}

\section{The origin of European studies on mortality in schizophrenia}

Studies on mortality in schizophrenia were conducted only in Northern and Western European countries: Denmark (7), United Kingdom (7), Finland (5), Sweden (2), Norway (1) and Netherlands (1). One of the studies was conducted in three Nordic countries: Denmark, Finland and Sweden, using common data register (Laursen et al. 2013). Two international studies were sponsored by pharmacological companies (Fleischhacker et al. 2014, Mittoux et al. 2013). No publication originally from Central and Eastern or Southern European countries met the inclusion criteria.

\section{Methods used in studies}

\section{Study characteristics}

In Danish, Finnish and one Swedish (Crump et al. 2013) studies data was obtained from national cohorts with a relevant diagnosis, within a specified period, except in the study by Schmidt et al. (2011), where the group was recruited from hospital inpatients. In other studies on schizophrenia cohorts represented country (Vinogradova et al. 2010, Tenback et al. 2012), region or populations of selected hospitals (Høye et al. 2011, Schoepf et al. 2012). Apart from schizophrenia, they also analysed mortality in people diagnosed with: substance abuse (Chang et al. 2010, Schmidt et al. 2011), affective disorder (Laursen et al. 2009, Chang et al. 2010, Laursen et al. 2011, Laursen 2011, Hoang et al. 2011, Hoang et al. 2013), schizoaffective disorder (Laursen et al. 2009, Rantanen et al. 2009, Tiihonen et al. 2009, Høye et al. 2011, Chang et al. 2010, Hoang et al. 2011, Schoepf et al. 2012, Hoang et al. 2013), and other psychoses with somatic comorbidities (Laursen et al. 2011).

\section{Medical data records}

The studies used national, local, hospital (Schoepf et al. 2012), university (Høye et al. 2011) and insurance agency (Tenback et al. 2012) medical data records. An access to data from various records for the same patients was possible due to ID numbers and record linkage systems. 


\section{Global risk factors influencing \\ mortality in schizophrenia}

WHO identify heavy smoking, obesity, lower level of physical activity, hyperglycemia, hyperlipidemia and hypertension as global mortality risk factors (WHO 2009) as well as modifiable life-style and health factors that contribute to the premature mortality in persons with schizophrenia (Yasamy et al. 2016). However, the majority of the studies failed to assess the influence of these conditions on mortality in schizophrenia: none of the studies took all the aforementioned risk factors into account, while the authors of only 7 of the papers considered at least one of them in their analyses. Three papers addressed hypertension and hyperlipidemia as specific risk factors (Schoepf et al. 2012, Crump et al. 2013, Laursen et al. 2013), 5 papers addressed smoking (Brown et al. 2010, Brown et al. 2012, Crump et al. 2013, Suvisaari et al. 2013, Fleischhacker et al. 2014) and 4 targeted hyperglycemia or diabetes (Schoepf et al. 2012, Crump et al. 2013, Laursen et al. 2013, Suvisaari et al. 2013). Only one study addressed physical inactivity (Brown et al. 2012) and none took obesity into account as a specific risk factor.

\section{Methodological limitations}

Diagnoses in the studies referred to classification systems from ICD-8 to ICD-10; however, according to Hjortso et al. (1989), there is a sufficient correlation of schizophrenia diagnostic criteria between successive editions. In some cases, verification of dual diagnosis was difficult to perform (Talaslahti et al. 2012, Tiihonen et al. 2012).

Cohorts in individual studies were not uniform. Some studies referred to hospitalised patients (Laursen et al. 2009, Hoang et al. 2011), while other concerned less severely ill patients treated in the primary care setting (Vinogradova et al. 2010). In addition, the frequency of visits at health care centres varied across studies (Chang et al. 2010, Schmidt et al. 2011). A follow-up period varied between studies, but none was continued until death of all subjects in the group.

Routinely collected data was incomplete. It did not include for example cause of death, diagnosis, information on disease course, received medications, or risk factors (Rantanen et al. 2009, Chang et al. 2010, Hoang et al. 2011, Schmidt et al. 2011, Tenback et al. 2012, Hoang et al. 2013). Also somatic comorbidities were difficult to confirm, as some patients died before disease developed (Vinogradova et al. 2010), or those were not diagnosed correctly (Schoepf et al. 2012). Not all studies considered the influence of regularly taken medications on a mortality rate (Vinogradova et al. 2010, Tenback et al. 2012, Tiihonen et al. 2012, Schoepf et al. 2012). Methodological details for analysed studies were presented in Table 1.

Table 1. Methodological characteristics of the studies comprised in the review

\begin{tabular}{|c|c|c|c|c|c|c|c|}
\hline Study & Nation, area & $\begin{array}{l}\text { Sample, case } \\
\text { ascertainment, } \\
\text { Methods }\end{array}$ & Diagnosis & $\begin{array}{l}\text { Period } \\
\text { (years } \\
\text { follow up) }\end{array}$ & $\begin{array}{l}\text { Size of } \\
\text { cohort } \\
(\mathrm{P}, \mathrm{M}, \mathrm{F})\end{array}$ & $\begin{array}{l}\text { Age of } \\
\text { respondents }\end{array}$ & $\begin{array}{l}\text { Observed death } \\
(\mathrm{P}, \mathrm{M}, \mathrm{F})\end{array}$ \\
\hline $\begin{array}{l}\text { Mittoux } \\
\text { et al. } 2013\end{array}$ & Denmark & register & N/A & $2002-2008$ & $\begin{array}{l}\text { P: } 9809 \\
- \\
-\end{array}$ & $\begin{array}{l}38.3 \\
(18.0-84.6)\end{array}$ & $\begin{array}{l}\text { P: } 175 \text { (1.8\%) } \\
- \\
-\end{array}$ \\
\hline $\begin{array}{l}\text { Laursen } \\
\text { et al. } 2011\end{array}$ & Denmark & $\begin{array}{l}\text { cohort, } \\
\text { ICD8/10 }\end{array}$ & $\begin{array}{l}\text { SCH, BP, } \\
\text { OTHER }\end{array}$ & 1995-2007 & $\begin{array}{l}\text { P: } 16079 \\
- \\
-\end{array}$ & N/A & N/A \\
\hline $\begin{array}{l}\text { Nielsen } \\
\text { et al. } 2013\end{array}$ & Denmark & $\begin{array}{l}\text { register, } \\
\text { ICD8/10 }\end{array}$ & $\mathrm{SCH}$ & $1980-2010$ & $\begin{array}{l}\text { P: } 14974 \\
\text { M: } 8148 \\
\text { F: } 6826\end{array}$ & N/A & $\begin{array}{l}\text { P: } 14974(100 \%) \\
\text { M: } 8148(100 \%) \\
\text { F: } 6826(100 \%)\end{array}$ \\
\hline Laursen 2011 & Denmark & $\begin{array}{l}\text { cohort, } \\
\text { ICD8/10 }\end{array}$ & $\mathrm{SCH}, \mathrm{BP}$ & 2000-2006 & $\begin{array}{l}\text { P: } 31727 \\
- \\
-\end{array}$ & N/A & $\begin{array}{l}\text { P: } 4247 \text { (13.4\%) } \\
- \\
-\end{array}$ \\
\hline $\begin{array}{l}\text { Laursen } \\
\text { et al. } 2009\end{array}$ & Denmark & $\begin{array}{l}\text { cohort, } \\
\text { ICD8/10 }\end{array}$ & $\begin{array}{l}\text { SCH, } \\
\text { BP, AP }\end{array}$ & 1994-2007 & $\begin{array}{l}\text { P: } 4592803 \\
- \\
-\end{array}$ & N/A & N/A \\
\hline $\begin{array}{l}\text { Schmidt } \\
\text { et al. } 2011\end{array}$ & $\begin{array}{l}\text { Denmark, St. Hans } \\
\text { Psychiatric Hospital } \\
\text { in Roskilde }\end{array}$ & cohort & N/A & 1993-2008 & $\begin{array}{l}\text { P: } 226 \\
\text { M: } 166 \\
\text { F: } 60\end{array}$ & N/A & $\begin{array}{l}\text { P: } 68 \text { (30.1\%) } \\
- \\
-\end{array}$ \\
\hline $\begin{array}{l}\text { Laursen } \\
\text { et al. } 2013\end{array}$ & Denmark & $\begin{array}{l}\text { cohort, } \\
\text { ICD8/10 }\end{array}$ & $\mathrm{SCH}, \mathrm{BP}$ & 1995-2008 & N/A & N/A & N/A \\
\hline $\begin{array}{l}\text { Нøye } \\
\text { et al. } 2011\end{array}$ & $\begin{array}{l}\text { Norway, Troms, } \\
\text { Finnmark }\end{array}$ & $\begin{array}{l}\text { cohort, } \\
\text { ICD8/9/10 }\end{array}$ & $\begin{array}{l}\text { SCH, AP, } \\
\text { OTHER }\end{array}$ & 1980-2006 & $\begin{array}{l}\text { P: } 1111 \\
\text { M: } 685 \\
\text { F: } 426\end{array}$ & N/A & $\begin{array}{l}\text { P: } 295(26.6 \%) \\
\text { M: } 192(28 . \%) \\
\text { F: } 103(24.2 \%)\end{array}$ \\
\hline
\end{tabular}

SCH - schizophrenia; $\mathrm{BP}$ - bipolar disorder; OP - other psychoses; AP - affective psychoses; OTHER - other psychiatric disorders; MR - mortality rate; MRR - mortality rate ratio; SMR - standardized mortality ratio; $\mathrm{M}$ - males; F - females; $\mathrm{P}$ - cohort size overall (males and females) 
Table 1. Continues

\begin{tabular}{|c|c|c|c|c|c|c|c|}
\hline Study & Nation, area & $\begin{array}{l}\text { Sample, case } \\
\text { ascertainment, } \\
\text { Methods } \\
\end{array}$ & Diagnosis & $\begin{array}{l}\text { Period } \\
\text { (years } \\
\text { follow up) }\end{array}$ & $\begin{array}{l}\text { Size of } \\
\text { cohort } \\
(\mathrm{P}, \mathrm{M}, \mathrm{F}) \\
\end{array}$ & $\begin{array}{l}\text { Age of } \\
\text { respondents }\end{array}$ & $\begin{array}{l}\text { Observed death } \\
(\mathrm{P}, \mathrm{M}, \mathrm{F})\end{array}$ \\
\hline $\begin{array}{l}\text { Tiihonen } \\
\text { et al. } 2009\end{array}$ & Finland & $\begin{array}{l}\text { cohort, } \\
\text { ICD8/9/10 }\end{array}$ & $\begin{array}{l}\text { SCH, AP, } \\
\text { OTHER }\end{array}$ & 1996-2006 & $\begin{array}{l}\text { P: } 66881 \\
\text { M: } 30803 \\
\text { F: } 36078\end{array}$ & 51 & $\begin{array}{l}\text { P: } 19735(29.5 \%) \\
\text { M: } 8323(27.0 \%) \\
\text { F: } 11412(37.6 \%)\end{array}$ \\
\hline $\begin{array}{l}\text { Rantanen } \\
\text { et al. } 2009\end{array}$ & Finland & $\begin{array}{l}\text { cohort, } \\
\text { ICD8/9/10 }\end{array}$ & SCH, AP & 1980-2003 & $\begin{array}{l}\text { P: } 23959 \\
- \\
-\end{array}$ & N/A & $\begin{array}{l}\text { P: } 1926(8.0 \%) \\
\text { M: } 1292(10.1 \%) \\
\text { F: } 634(5.7 \%)\end{array}$ \\
\hline $\begin{array}{l}\text { Talaslahti } \\
\text { et al. } 2012\end{array}$ & Finland & $\begin{array}{l}\text { cohort, } \\
\text { ICD8/9/10 }\end{array}$ & SCH, AP & 1969-2008 & $\begin{array}{l}\text { P: } 9461 \\
\text { M: } 2957 \\
\text { F: } 6504\end{array}$ & N/A & $\begin{array}{l}\text { P: } 5596(59 \%) \\
- \\
-\end{array}$ \\
\hline $\begin{array}{l}\text { Suvisaari } \\
\text { et al. } 2013\end{array}$ & Finland & cohort & N/A & 2000-2009 & $\begin{array}{l}\text { P: } 67 \\
\text { M: } 30803 \\
\text { F: } 37\end{array}$ & 53.7 & $\begin{array}{l}\text { P: } 18(26.9 \%) \\
- \\
-\end{array}$ \\
\hline $\begin{array}{l}\text { Tiihonen } \\
\text { et al. } 2012\end{array}$ & Finland & $\begin{array}{l}\text { register, } \\
\text { ICD10 }\end{array}$ & $\mathrm{SCH}$ & $2000-2007$ & $\begin{array}{l}\text { P: } 2588 \\
\text { M: } 1604 \\
\text { F: } 984\end{array}$ & 37.8 & $\begin{array}{l}\text { P: } 160(4.0 \%) \\
- \\
-\end{array}$ \\
\hline $\begin{array}{l}\text { Chang } \\
\text { et al. } 2010\end{array}$ & $\begin{array}{l}\text { G. Britain, Lambeth, } \\
\text { Southwark, Lewisham, } \\
\text { Croydon }\end{array}$ & $\begin{array}{l}\text { cohort, } \\
\text { ICD10 }\end{array}$ & $\begin{array}{l}\text { SCH, BP, } \\
\text { AP, OTHER }\end{array}$ & 2007-2009 & $\begin{array}{l}\text { P: } 31719 \\
- \\
-\end{array}$ & N/A & N/A \\
\hline $\begin{array}{l}\text { Hoang } \\
\text { et al. } 2013\end{array}$ & $\begin{array}{l}\text { G. Britain, Lambeth, } \\
\text { Southwark, Lewisham, } \\
\text { Croydon }\end{array}$ & cohort, ICD10 & $\begin{array}{l}\text { SCH, BP, } \\
\mathrm{AP}, \mathrm{OP}\end{array}$ & 2006-2008 & $\begin{array}{l}\text { P: } 37607 \\
- \\
-\end{array}$ & N/A & $\begin{array}{l}\text { P: } 480(1.3 \%) \\
- \\
-\end{array}$ \\
\hline $\begin{array}{l}\text { Vinogradova } \\
\text { et al. } 2010\end{array}$ & G. Britain & $\begin{array}{l}\text { cohort, } \\
\text { GP computer } \\
\text { codes }\end{array}$ & N/A & 2000-2005 & $\begin{array}{l}\text { P: } 257 \\
\text { M: } 116 \\
\text { F: } 141\end{array}$ & $60.9 \pm 12.5$ & N/A \\
\hline $\begin{array}{l}\text { Hoang } \\
\text { et al. } 2011\end{array}$ & G. Britain & $\begin{array}{l}\text { record linkage, } \\
\text { ICD10 }\end{array}$ & $\begin{array}{l}\text { SCH, BP, } \\
\mathrm{AP}, \mathrm{OP}\end{array}$ & 1999-2007 & N/A & N/A & N/A \\
\hline $\begin{array}{l}\text { Brown } \\
\text { et al. } 2012\end{array}$ & G. Britain & cohort & N/A & 1996-2006 & $\begin{array}{l}\text { P: } 95 \\
\text { M: } 48 \\
\text { F: } 47\end{array}$ & 54.3 & $\begin{array}{l}\text { P: } 29(30.5 \%) \\
- \\
-\end{array}$ \\
\hline $\begin{array}{l}\text { Brown } \\
\text { et al. } 2010\end{array}$ & $\begin{array}{l}\text { G. Britain, } \\
\text { Southampton }\end{array}$ & record linkage & N/A & 1981-2006 & $\begin{array}{l}\text { P: } 370 \\
\text { M: } 213 \\
\text { F: } 157\end{array}$ & $\begin{array}{l}\text { M-64.4 } \\
\text { F-67.3 }\end{array}$ & $\begin{array}{l}\text { P: } 164(44.3 \%) \\
\text { M: } 92(43.2 \%) \\
\text { F: } 72(45.9 \%)\end{array}$ \\
\hline $\begin{array}{l}\text { Schoepf } \\
\text { et al. } 2012\end{array}$ & $\begin{array}{l}\text { G. Britain, Univer } \\
\text { sity Hospital Bir- } \\
\text { mingham NHS Trust }\end{array}$ & $\begin{array}{l}\text { case- control, } \\
\text { ICD10 }\end{array}$ & $\mathrm{SCH}$ & $2000-2007$ & $\begin{array}{l}\text { P: } 679 \\
\text { M: } 241 \\
\text { F: } 438\end{array}$ & $54.4 \pm 17.7$ & $\begin{array}{l}\text { P: } 100(14.7 \%) \\
\text { M: } 58(24.1 \%) \\
\text { F: } 42(9.6 \%)\end{array}$ \\
\hline $\begin{array}{l}\text { Tenback } \\
\text { et al. } 2012\end{array}$ & Netherlands & cohort & N/A & 2006-2008 & $\begin{array}{l}\text { P: } 7415 \\
\text { M: } 4538 \\
\text { F: } 2877\end{array}$ & N/A & N/A \\
\hline $\begin{array}{l}\text { Crump } \\
\text { et al. } 2013\end{array}$ & Sweden & cohort, ICD10 & $\mathrm{SCH}$ & 2003-2009 & $\begin{array}{l}\text { P: } 8277 \\
\text { M: } 4787 \\
\text { F: } 3490\end{array}$ & N/A & $\begin{array}{l}\text { P: } 1121(13.5 \%) \\
\text { M: } 603(12.6 \%) \\
\text { F: } 518(14.8 \%)\end{array}$ \\
\hline $\begin{array}{l}\text { Tabita } \\
\text { et al. } 2012\end{array}$ & $\begin{array}{l}\text { Sweden, Örebro } \\
\text { County }\end{array}$ & cohort & N/A & 1992-2008 & $\begin{array}{l}\text { P: } 28 \\
\text { M: } 25 \\
\text { F: } 3\end{array}$ & N/A & N/A \\
\hline Laursen 2013 & $\begin{array}{l}\text { Denmark, Finland, } \\
\text { Sweden }\end{array}$ & $\begin{array}{l}\text { register, } \\
\text { ICD8/9/10 }\end{array}$ & SCH, BP & 2000-2007 & $\begin{array}{l}\text { P: } 66088 \\
- \\
-\end{array}$ & N/A & $\begin{array}{l}\text { P: } 9535(14.4 \%) \\
- \\
-\end{array}$ \\
\hline $\begin{array}{l}\text { Fleischhacker } \\
\text { et al. } 2014\end{array}$ & 18 countries & $\begin{array}{l}\text { dgn by } \\
\text { physician }\end{array}$ & $\mathrm{SCH}$ & 2002-2008 & $\begin{array}{l}\text { P: } 18154 \\
\text { M: } 9761 \\
\text { F: } 8393\end{array}$ & N/A & $\begin{array}{l}\text { P: } 35(0.2 \%) \\
\text { M: } 19(0.2 \%) \\
\text { F: } 16(0.2 \%)\end{array}$ \\
\hline $\begin{array}{l}\text { Castagnini } \\
\text { et al. } 2013\end{array}$ & Denmark & register, ICD10 & $\begin{array}{l}\mathrm{SCH}, \mathrm{BP}, \\
\mathrm{OP}\end{array}$ & 1995-20013 & $\begin{array}{l}P: 4576 \\
- \\
-\end{array}$ & $32.6 \pm 11.5$ & $\begin{array}{l}P: 233(5.1 \%) \\
- \\
-\end{array}$ \\
\hline
\end{tabular}

SCH - schizophrenia; BP - bipolar disorder; OP - other psychoses; AP - affective psychoses; OTHER - other psychiatric disorders; MR - mortality rate; MRR - mortality rate ratio; SMR - standardized mortality ratio; M - males; F - females; $\mathrm{P}$ - cohort size overall (males and females) 
Table 2. The percentage of the main causes of death in the analysed studies (where available)

\begin{tabular}{|c|c|c|c|c|c|c|c|c|c|}
\hline Study & $\begin{array}{l}\text { Observed } \\
\text { death } \\
(\mathrm{P}, \mathrm{M}, \mathrm{F})\end{array}$ & $\begin{array}{l}\text { Suicide } \\
(\mathrm{P}, \mathrm{M}, \mathrm{F})\end{array}$ & $\begin{array}{l}\text { Neoplasm } \\
(\mathrm{P}, \mathrm{M}, \mathrm{F})\end{array}$ & $\begin{array}{l}\text { Circulatory } \\
\text { diseases }\end{array}$ & $\begin{array}{r}\text { Cau } \\
\text { Infection }\end{array}$ & $\begin{array}{l}\text { se of Death } \\
\text { Endocrine diseases } \\
\text { incl. diabetes \& } \\
\text { Metabolic diseases }\end{array}$ & $\begin{array}{l}\text { Respi- } \\
\text { ratory } \\
\text { diseases }\end{array}$ & $\begin{array}{l}\text { Nervous } \\
\text { diseases }\end{array}$ & $\begin{array}{l}\text { Digestive } \\
\text { system } \\
\text { diseases }\end{array}$ \\
\hline $\begin{array}{l}\text { Mittoux } \\
\text { et al. } 2013\end{array}$ & $\begin{array}{l}\text { P: } 175 \\
- \\
-\end{array}$ & N/A & N/A & N/A & N/A & N/A & N/A & N/A & N/A \\
\hline $\begin{array}{l}\text { Laursen } \\
\text { et al. } 2011\end{array}$ & N/A & N/A & N/A & N/A & N/A & N/A & N/A & N/A & N/A \\
\hline $\begin{array}{l}\text { Nielsen } \\
\text { et al. } 2013\end{array}$ & $\begin{array}{l}\text { P: } 14974 \\
\text { M: } 8148 \\
\text { F: } 6826\end{array}$ & $\begin{array}{l}\text { P: } 10.3 \% \\
- \\
-\end{array}$ & P: $11.6 \%$ & $24.3 \%$ & $11.45 \%$ & $2.1 \%$ & $3.9 \%$ & $1 \%$ & N/A \\
\hline Laursen 2011 & $\begin{array}{l}P: 4247 \\
- \\
-\end{array}$ & N/A & N/A & $22.6 \%$ & N/A & N/A & N/A & N/A & N/A \\
\hline $\begin{array}{l}\text { Laursen } \\
\text { et al. } 2009\end{array}$ & N/A & N/A & N/A & N/A & N/A & N/A & N/A & N/A & N/A \\
\hline $\begin{array}{l}\text { Schmidt } \\
\text { et al. } 2011\end{array}$ & $\begin{array}{l}\text { P: } 68 \\
- \\
-\end{array}$ & $\begin{array}{l}P: 15-22 \% \\
- \\
-\end{array}$ & N/A & N/A & N/A & N/A & N/A & N/A & N/A \\
\hline $\begin{array}{l}\text { Laursen } \\
\text { et al. } 2013\end{array}$ & N/A & N/A & N/A & N/A & N/A & N/A & N/A & N/A & N/A \\
\hline $\begin{array}{l}\text { Høye } \\
\text { et al. } 2011\end{array}$ & $\begin{array}{l}\text { P: } 295 \\
\text { M: } 192 \\
F: 103\end{array}$ & $\begin{array}{l}\text { P: } 16.6 \% \\
\text { M: } 20.6 \% \\
\text { F: } 8.7 \%\end{array}$ & $\begin{array}{l}\text { P: } 14.9 \% \\
\text { M: } 14.6 \% \\
\text { F: } 15.5 \%\end{array}$ & $33.9 \%$ & N/A & N/A & N/A & N/A & N/A \\
\hline $\begin{array}{l}\text { Tiihonen } \\
\text { et al. } 2009\end{array}$ & $\begin{array}{l}\text { P: } 19735 \\
\text { M: } 8323 \\
\text { F: } 11412\end{array}$ & N/A & N/A & N/A & N/A & N/A & N/A & N/A & N/A \\
\hline $\begin{array}{l}\text { Rantanen } \\
\text { et al. } 2009\end{array}$ & $\begin{array}{l}\text { P: } 1926 \\
\text { M: } 1292 \\
\text { F: } 634\end{array}$ & $\begin{array}{l}\text { P: } 42.0 \% \\
\text { M: } 44.5 \% \\
\text { F: } 36.9 \%\end{array}$ & $\begin{array}{l}\text { P: } 6.7 \% \\
- \\
-\end{array}$ & $20.9 \%$ & N/A & $7.1 \%$ & $3.9 \%$ & N/A & N/A \\
\hline $\begin{array}{l}\text { Talaslahti } \\
\text { et al. } 2012\end{array}$ & $\begin{array}{l}\text { P: } 5596 \\
- \\
-\end{array}$ & $\begin{array}{l}\text { P: } 0.8 \% \\
- \\
-\end{array}$ & $\begin{array}{l}\text { P: } 14.7 \% \\
- \\
-\end{array}$ & $42.7 \%$ & $1 \%$ & $1.5 \%$ & $12.4 \%$ & $10.5 \%$ & $3.7 \%$ \\
\hline $\begin{array}{l}\text { Suvisaari } \\
\text { et al. } 2013\end{array}$ & $\begin{array}{l}\mathrm{P}: 18 \\
- \\
-\end{array}$ & N/A & $\begin{array}{l}\mathrm{P}: 22.2 \% \\
- \\
-\end{array}$ & $33.3 \%$ & N/A & N/A & N/A & N/A & N/A \\
\hline $\begin{array}{l}\text { Tiihonen } \\
\text { et al. } 2012\end{array}$ & $\begin{array}{l}\text { P: } 160 \\
- \\
-\end{array}$ & $\begin{array}{l}\text { P: } 21.9 \% \\
- \\
-\end{array}$ & $\begin{array}{l}\text { P: } 18.8 \% \\
- \\
-\end{array}$ & $21.9 \%$ & $1.3 \%$ & $2.5 \%$ & $4.4 \%$ & $1.9 \%$ & $5.6 \%$ \\
\hline $\begin{array}{l}\text { Chang } \\
\text { et al. } 2010\end{array}$ & N/A & N/A & N/A & N/A & N/A & N/A & N/A & N/A & N/A \\
\hline $\begin{array}{l}\text { Hoang } \\
\text { et al. } 2013\end{array}$ & $\begin{array}{l}\text { P: } 480 \\
- \\
-\end{array}$ & N/A & N/A & N/A & N/A & N/A & N/A & N/A & N/A \\
\hline $\begin{array}{l}\text { Vinogradova } \\
\text { et al. } 2010\end{array}$ & N/A & N/A & N/A & N/A & N/A & N/A & N/A & N/A & N/A \\
\hline $\begin{array}{l}\text { Hoang } \\
\text { et al. } 2011\end{array}$ & N/A & N/A & N/A & N/A & N/A & N/A & N/A & N/A & N/A \\
\hline $\begin{array}{l}\text { Brown } \\
\text { et al. } 2012\end{array}$ & $\begin{array}{l}\text { P: } 29 \\
- \\
-\end{array}$ & $\begin{array}{l}\mathrm{P}: 0 \% \\
- \\
-\end{array}$ & $\begin{array}{l}\mathrm{P}: 31 \% \\
- \\
-\end{array}$ & $17.2 \%$ & $3.4 \%$ & N/A & $31 \%$ & $13.8 \%$ & N/A \\
\hline $\begin{array}{l}\text { Brown } \\
\text { et al. } 2010\end{array}$ & $\begin{array}{l}\text { P: } 164 \\
\text { M: } 92 \\
\text { F: } 72\end{array}$ & $\begin{array}{l}\text { P: } 8.5 \% \\
- \\
-\end{array}$ & $\begin{array}{l}\text { P: } 18.3 \% \\
\text { M: } 21.7 \% \\
\text { F: } 13.4 \%\end{array}$ & $34.8 \%$ & N/A & $4.3 \%$ & $15.9 \%$ & $3 \%$ & $4.3 \%$ \\
\hline $\begin{array}{l}\text { Schoepf } \\
\text { et al. } 2012\end{array}$ & $\begin{array}{l}\text { P: } 100 \\
\text { M: } 58 \\
\text { F: } 42\end{array}$ & N/A & N/A & N/A & N/A & N/A & N/A & N/A & N/A \\
\hline $\begin{array}{l}\text { Tenback } \\
\text { et al. } 2012 \\
\end{array}$ & N/A & N/A & N/A & N/A & N/A & N/A & N/A & N/A & N/A \\
\hline
\end{tabular}


Table 2. Continues

\begin{tabular}{|c|c|c|c|c|c|c|c|c|c|}
\hline Study & $\begin{array}{l}\text { Observed } \\
\text { death } \\
(\mathrm{P}, \mathrm{M}, \mathrm{F})\end{array}$ & $\begin{array}{l}\text { Suicide } \\
(\mathrm{P}, \mathrm{M}, \mathrm{F})\end{array}$ & $\begin{array}{l}\text { Neoplasm } \\
(\mathrm{P}, \mathrm{M}, \mathrm{F})\end{array}$ & $\begin{array}{l}\text { Circulatory } \\
\text { diseases }\end{array}$ & $\begin{array}{r}\text { Cau } \\
\text { Infection }\end{array}$ & $\begin{array}{l}\text { se of Death } \\
\text { Endocrine diseases } \\
\text { incl. diabetes \& } \\
\text { Metabolic diseases }\end{array}$ & $\begin{array}{l}\text { Respi- } \\
\text { ratory } \\
\text { diseases }\end{array}$ & $\begin{array}{l}\text { Nervous } \\
\text { diseases }\end{array}$ & $\begin{array}{l}\text { Digestive } \\
\text { system } \\
\text { diseases }\end{array}$ \\
\hline $\begin{array}{l}\text { Crump } \\
\text { et al. } 2013\end{array}$ & $\begin{array}{l}\mathrm{P}: 1121 \\
\mathrm{M}: 603 \\
\mathrm{~F}: 518\end{array}$ & $\begin{array}{l}\text { P: } 5.7 \% \\
\text { M: } 7.6 \% \\
\text { F: } 3.5 \%\end{array}$ & $\begin{array}{l}\text { P: } 14,7 \% \\
\text { M: } 12.8 \% \\
\text { F: } 17.0 \%\end{array}$ & $56.5 \%$ & N/A & $3.5 \%$ & N/A & N/A & N/A \\
\hline $\begin{array}{l}\text { Tabita } \\
\text { et al. } 2012\end{array}$ & N/A & N/A & N/A & N/A & N/A & N/A & N/A & N/A & N/A \\
\hline Laursen 2013 & $\begin{array}{l}\text { P: } 9535 \\
- \\
-\end{array}$ & N/A & N/A & N/A & N/A & N/A & N/A & $0.5 \%$ & N/A \\
\hline $\begin{array}{l}\text { Fleischhacker } \\
\text { et al. } 2014\end{array}$ & $\begin{array}{l}\text { P: } 35 \\
\text { M: } 19 \\
\text { F: } 16\end{array}$ & $\begin{array}{l}\text { P: } 0.2 \% \\
\text { M: } 0.2 \% \\
\text { F: } 0,2 \%\end{array}$ & N/A & N/A & N/A & N/A & N/A & N/A & N/A \\
\hline $\begin{array}{l}\text { Castagnini } \\
\text { et al. } 2013\end{array}$ & $\begin{array}{l}\text { P: } 233 \\
- \\
-\end{array}$ & P: $31.8 \%$ & $\begin{array}{l}\text { P: } 18.75 \% \\
- \\
-\end{array}$ & $19.6 \%$ & $0.9 \%$ & $4.5 \%$ & $8.9 \%$ & $4.5 \%$ & $10.7 \%$ \\
\hline
\end{tabular}

\section{Main causes of death in patients with schizophrenia}

\section{Natural and unnatural causes of death}

Schizophrenia patients were characterised by higher mortality in comparison with the general population (Auquier et al. 2007, Nielsen et al. 2010, Tenback et al. 2012, Suvisaari et al. 2013), with natural causes prevailing over external ones (Auquier et al. 2007, Brown et al. 2010, Laursen et al. 2011, Laursen 2011, Crump et al. 2013), and with chronic disease-related mortality rate ratio (MRR) ranging from 4.64 to 7.10 for men, and from 3.03 to 6.06 for women, depending on calculation method (Laursen et al. 2011). In the Danish study, unnatural causes slightly prevailed over natural ones, causing $51.9 \%$ of deaths (Castagnini et al. 2013). Also standardized mortality ratio (SMR) for deaths for external causes was higher than the rate for natural causes (SMR 8.1; 95\% CI 6.8-9.7 vs SMR 3.2; 95\% CI 2.6-3.8, respectively) (Castagnini et al. 2013). In Finnish studies on patients with the first schizophrenia episode, suicide was also the most common cause of death during 5-year observations from 1980-1998 and 1995-2001 (Rantanen et al. 2009). The percentage of the main causes of death in each study, where available, was presented in Table 2.

\section{Cardiovascular diseases}

Brown et al. (2010) revealed that cardiovascular diseases had the highest effect on mortality increase, representing one third of all deaths, with SMR of 2.58 (95\% CI: 1.95-3.34). In Sweden, men and women died of ischaemic heart disease 14.5 and 12.7 years earlier than people in the reference population, respectively (Crump et al. 2013). In Denmark, the frequency of medical interventions for heart diseases was slightly higher (incidence rate ratio=1.04, 95\% CI, 1.00-1.08), whereas mortality rate was significantly higher (MRR=3.52, 95\% CI, 3.22-3.84) in patients with schizophrenia versus the general population (Laursen et al. 2009). Also in Denmark, a study on a large sample showed that persons with schizophrenia are characterised by an all-cause MRR=2.37 (95\% CI, 2.13-2.64) versus population with no psychiatric contacts when there was a previous diagnosis of myocardial infarct or cerebrovascular disease in either group. Even higher allcause MRR=5.67 (95\% CI, 5.23-6.15) was found when there was no prior history of myocardial infarct or cerebrovascular disease, analogically (Laursen et al. 2014). MRR for cardiac death in schizophrenia was calculated at 2.67 (95\% CI 1.82-3.94) with no previous myocardial infarct or cerebrovascular disease and at 2.96 (95\% CI 2.26-3.98) with either of the diseases diagnosed in the past, compared to population with no psychiatric contacts (Laursen et al. 2014).

The Swedish study showed that schizophrenia patients who died of myocardial infarction were less often diagnosed with ischaemic heart disease than people dying for the same reasons in the general population (Crump et al. 2013). Scandinavian studies showed that in Finland and Denmark in schizophrenia patients, SMR associated with cardiovascular diseases was lower than all causes of death in total. However, when ischaemic heart disease, cerebrovascular disease and other cardiovascular diseases (including rheumatic heart disease, hypertension, arterial or venous diseases) were considered separately, no significant SMR deviations were demonstrated in Denmark and Sweden, while in Finland that rate was higher for other cardiovascular diseases (Laursen et al. 2013).

\section{Cancers}

Crump et al. (2013) demonstrated that malignant cancers were less often diagnosed in schizophrenia patients dying of cancer in comparison with those dying of the same cause in the general population. In the British prospective study of 370 patients from 1981/822006, cancers were the second cause of death, after cardiovascular diseases (30 and 56 deaths, respectively), with a total SMR of 1.49 (95\% CI, 1.00-2.12). In this 
study gender-specific SMR was estimated at $1.93(95 \%$ CI, 1.18-2.98) for men and 1.02 (95\% CI, 0.49-1/88) for women. The most common cancers affected lungs (13 cases) and breast ( 4 cases) (Brown et al. 2010).

\section{Diabetes}

The same study (Brown et al. 2010) showed a significantly higher SMR for diabetes in schizophrenia patients: 6.14 (95\%CI, 1.67-15.73). Type 2 diabetes was also more frequent in comparison with all hospitalised patients. Type 2 diabetes was the most common disease in schizophrenia patients who died during 7year observations, representing $24 \%$ of this group, versus $10.5 \%$ of deaths in the control group, with $\mathrm{RR}=2.2$ (Schoepf et al. 2012). In another study (2000-2005), the risk (HR) for patients diagnosed with schizophrenia and diabetes was 1.52 (95\%CI 1.17-1.97) versus patients with diabetes only (regardless of diabetes type, after adjustment for age of diabetes diagnosis, gender, smoking status, deprivation, obesity and the use of statins) (Vinogradova et al. 2010).

\section{Suicides}

In the Danish study, suicide was the most commonly specified cause of death (31.8\%), also characterised by the highest SMR: 15.2 (95\%CI 12.1-19.1) (Castagnini et al. 2013). Brown et al. (2010) noted even higher SMRs for suicide and external unspecified reasons that were estimated at 18.18 (95\%CI, 9.94-30.51) and 9.28 (95\%CI, 1.91-27.13), respectively. In the Swedish study, an increase in the mortality rate in schizophrenia was 6 times higher for women and 4.4 times higher for men (Crump et al. 2013). In the Finnish study the highest risk of death by suicide in schizophrenia was found in the youngest group of patients (15-29 years) (Rantanen et al. 2009).

\section{Psychopharmacotherapy}

Despite a significant increase in use of second-generation antipsychotics (SGA) and concerns about their negative effect on the mortality rate resulting from deteriorated metabolic parameters, the FIN11 study showed that the mortality gap did not change significantly during 20 years (25 years in 1996 and 22.5 years in 2006) (Tiihonen et al. 2009). It was also found that a long-term (7-11 years) use of any antipsychotics was related to lower mortality $(0.81,0.77-0.84)$, and regular treatment reduced the risk of death to $\mathrm{HR}=0.68$ (95\% CI 0.65-0.71) versus patients who did not receive pharmacological treatment. No increase in mortality caused by ischaemic heart disease was observed. The lowest mortality rate and a risk of suicide were associated with clozapine treatment (Tiihonen et al. 2009). The Finnish study showed that mortality did not increase when two or more antipsychotics were combined versus monotherapy (HR, 0.86; 95\% CI, 0.51-1.44); however, when they were combined with benzodiazepines, mortality increased in general (HR, 1.91; 95\% CI, 1.13-3.22), from suicide (HR, 3.83; 95\% CI, 1.45-10.12) and other causes (HR, 1.60; 95\% CI,
0.86-2.97) (Tiihonen et al. 2012). The use of antidepressants in schizophrenia patients was not associated with increased mortality (HR, 0.57; 95\% CI, 0.28-1.16), with lower suicide risk (HR, 0.15; 95\% CI, 0.03-0.77) (Tiihonen et al. 2012). When no antipsychotics were used, the mortality in schizophrenia patients increased significantly (HR, 2.09; 95\% CI, 1.34-3.26). HRs for suicide reached 0.87 (95\% CI, 0.32-2.34) in patients receiving two or more antipsychotics, 0.15 (95\% CI, $0.03-0.77$ ) in those receiving antidepressants, and 3.83 (95\% CI, 1.45-10.12) when benzodiazepines were used (Tiihonen et al. 2012). In a cohort observed for 3 years it was demonstrated that a risk of death is significantly elevated in patients taking first-generation antipsychotics (FGA) $(\mathrm{HR}=2.36)$ and mood stabilisers ( $\mathrm{HR}=8.42)$, while SGA significantly reduced the risk $(\mathrm{HR}=0.89)$ versus the whole population of schizophrenia patients (Tenback et al. 2012). In the Swedish study, an increased general risk of death was demonstrated for patients not receiving any antipsychotics (adjusted hazard ratio=1.45, 95\%CI=1.20-1.76), particularly cancer related mortality increased (adjusted hazard ratio $=1.94$, 95\%CI=1.13-3.32) (Crump et al. 2013). Although the Finnish study showed that any antipsychotics therapy was associated with increased general mortality (HR=2.34; 95\%CI=1.86-2.96) versus untreated people, yet treatment of people with psychotic disorder did not significantly increase a mortality risk $(\mathrm{HR}=1.08 ; 95 \%$ $\mathrm{CI}=0.58-2.00$ ) (Suvisaari et al. 2013).

\section{Age}

The Finnish study of Talaslahti et al. (2012) (9461 patients) showed that SMR in the patients aged over 65 years was 2.69 (95\%CI 2.62-2.76), reaching a statistically significant value in men - 3.00 (2.87-3.14) versus women - 2.55 (2.47-2.63) (RR 1.18, 95\%CI $1.11-1.24, \mathrm{p}<0.05)$. For natural causes of death, a total SMR was 2.58 (2.51-2.65; $n=5301), 2.87$ for men (2.74-3.00; $n=1832$ ), and 2.45 for women (2.37-2.53; $\mathrm{n}=3469$ ). For unnatural causes of death, a total SMR was 11.04 (9.75-12.47; n=262), 11.52 for men (9.3514.04; $n=98)$, and 10.78 for women (9.20-12.56; $n=164)$. The most common causes of death were the same as in the general population, with an increase in SMR for cardiovascular diseases - 1.89 (1.81-1.96) and cancers - 1.93 (1.80-2.07) demonstrated for schizophrenia. Falls were the most common unnatural cause of death, followed by suicides, with other accidents and their consequences being less common. SMR was higher in patients with at least one inpatient hospitalization in 5 years before being included in the study (3.92; 3.73-4.11) than in those not hospitalised in that period (2.37; 2.29-2.44) (Talaslahti et al. 2012). In the Finnish study by Rantanen et al., age was a risk factor for general mortality and mortality for natural causes in both sexes (Rantanen et al. 2009). Chang et al. found that standardised mortality rates are inversely associated with age (SMR for 15-44 years, 45-64 years and 65+ years was: 4.73 (3.52-6.22, $\mathrm{n}=51), 3.44$ (2.82-4.16, 
$\mathrm{n}=106)$, and $1.63(1.39-1.89, \mathrm{n}=165)$, respectively) (Chang et al. 2010). Similarly, the Danish study showed that in schizophrenia the highest SMR was associated with the age group. It was estimated at 15.1 (95\% CI 9.6-23.6) in patients aged 15-19 years and gradually decreased in older groups reaching 1.8 (95\% CI 0.74.2) in those aged 60-64 years (Castagnini et al. 2013).

\section{Sex}

In the British study, SMR for men with schizophrenia was higher than for women, amounting to: 2.78 (2.40-3.19, $\mathrm{n}=196)$ and $1.74(1.45-2.07, \mathrm{n}=126)$, respectively (Chang et al. 2010). The Norwegian study by Høye et al. (1980-2006) also showed that men are characterised by higher mortality than women, versus the general population with SMR: 3.5 (95\% CI: 3.1-4.1) and 2.6 (95\% CI: 2.1-3.2), respectively, and throughout the observation period values were higher for all specified causes of death (Høye et al. 2011). However, an increase in SMR was observed for women admitted to a hospital for the first time in 1993-2006 versus the previous period. SMR for both sexes was higher in patients admitted in 1998-2006 versus those admitted in 1980-1988 (Høye et al. 2011). In another Scandinavian study results from observations in 2000-2007 were unambiguous: SMR was 3.3, 3.0, and 2.9 for men, and 3.9, 2.7 and 2.9 for women in Finland, Denmark and Sweden, respectively (Laursen et al. 2013).

\section{Deinstitutionalisation}

During five years of observations in 30278 schizophrenia patients discharged from a hospital in 19801998, Rantanen et al. (2009) found a significant decrease in men's mortality from unnatural causes in 1995-1998 resulting from significant reduction in suicide rates versus 1980-1984. In subjects enrolled in the study in 1990-1994 a significant increase in mortality for unnatural causes other than suicide was noted. Similar trends were not found for women or for natural causes of death. However, suicides were the most common cause of death and represented $42 \%$ of cases. Interesting outcomes were provided by one of the international studies, according to which the increase in number of physicians by 1 per 10000 citizens results in the increase the risk of death among patients with schizophrenia in Europe by ca. 2\%. This pattern has not been replicated in Asia, however (Mittoux et al. 2013).

\section{Predictive factors}

In 10 years of observations, an increased risk of death for natural causes was related to active psychosis (HR 2.62, 95\% CI 1.13-6.07) on enrolment into a study (Brown et al. 2012). The risk of death was also significantly correlated with smoking (HR 2.53, 95\% CI 1.01-6.34) and age on inclusion in the study (HR 1.08, 95\% CI 1.02-1.13) for each additional year over 49.4 years (Brown et al. 2012). BMI, a sedentary lifestyle and a high consumption of saturated fatty acids were not significant predictors for death. However, a low consumption of unsaturated fatty acids, can indicate a tendency for increased mortality, although statistically insignificant. The Finnish study demonstrated following significant predictors of mortality: age ( $\mathrm{HR}=1.07 ; 95 \%$ $\mathrm{CI}=1.03-1.12)$, active smoking habit $(\mathrm{HR}=4.29 ; 95 \%$ $\mathrm{CI}=1.06-17.4)$ and type 2 diabetes ( $\mathrm{HR}=4.40 ; 95 \%$ CI $=$ 1.35-14.3) (Suvisaari et al. 2013).

\section{Smoking}

SMR was significantly higher in smokers (SMR=3.79 vs SMR=1.94), with more than double increase in mortality (Brown et al. 2010). The proportion of smokers with schizophrenia was more than double the percentage of smokers in the general population (73\% vs. $35 \%$ ), and the excess mortality resulted from smokingrelated diseases in 70\% of cases (Brown et al. 2010).

\section{Mortality trends in European studies}

Danish studies show that from 1980 to 2010, when in the general population an annual increase in an average life expectancy reached 0.28 years for men and 0.31 years for women on average, the average life expectancy in schizophrenia patients dropped by 0.04 and 0.05 years for men and women, respectively (Nielsen et al. 2010). Decrease in the average life expectancy in schizophrenia patients was even less favourable, of 0.24 and 0.19 in each calendar year, respectively, when "intentional self-harm" (ICD-10: X60-X84) was excluded as a cause of death, thus indicating that this unfavourable tendency also covers deaths for natural causes. In comparison to the general population, HR for mortality was: 2.27 (95\% CI: 2.202.33) for men and 1.88 (95\% CI, 1.82-1.93) for women (Nielsen et al. 2010).

Brown et al. (2010) revealed an increase in SMR in total in 1981-2006 (following an initial drop from 3.76 to 2.64 in 1981-1986), from 2.64 (95\% CI: $174-84$ ) to 2.92 (95\% CI: 212-392). This mainly resulted from an increase in mortality from natural causes, including an SMR increase for cardiovascular causes from 1.29 (1981-1986) to 3.50 (2001-2006), and a radical drop in SMR for unnatural causes from 24.82 to 4.72 in the same period.

Unfavourable trends were also observed in a Norwegian study conducted in 1980-2006 (Høye et al. 2011), demonstrating an increase in SMR during a 9year follow-up. For women included in the study during last 14 years, an increase in mortality gap (MG) was noted, versus a general female population in the same period.

Hoang et al. (2013) concluded that even if the health care availability to schizophrenia patients was improved, and suicide and open verdict cases prevention was the same as for a general population, the MG between schizophrenia patients and the general population would still exist. SMR would then drop from 4.23 (95\% CI: 85-4.60) to 2.38 (95\% CI: 2.09-2.66) in total, and from 4.55 (95\% CI: $4.05-5.05)$ to 2.48 (95\% CI: $2.11-2.85$ ) for men and from 3.68 (95\% CI: 3.11-4.24) to 2.16 
(95\% CI: 1.73-2.59) for women. Patients under 45 years of age would mostly benefit from elimination of deaths possible to avoid, as for them SMR would drop from 7.13 (95\% CI: 6.02-8.23) to 3.32 (95\% CI: 2.574.07); however, the mortality gap would not be completely eliminated.

In the Danish study (Laursen 2011), the life expectancy for schizophrenia patients is significantly shorter than for the general population, by 18.7 years for men and by 16.3 years for women, on average, with deaths of natural causes having a stronger effect on its shortening than deaths of external causes. In Sweden the noted difference in the life expectancy was 15 and 12 years, for men and women, respectively (Crump et al. 2013). Comorbid substance use disorders were associated with reduced life expectancy. During 15 years of observations, a mean age at death was 62.6 years for patients without substance use disorders and 49.2 for patients with dual diagnosis (Schmidt et al. 2011). In Denmark, Finland and Sweden, a difference in life expectancy (LE) for schizophrenia patients versus a general population in these countries was 20.0, 17.1, 18.9 years for men and 16.5, 15.6, 16.9 years for women, respectively (Laursen et al. 2013).

In 1999-2006 in UK a significant increase in SMR in schizophrenia occurred, from 1.6 (95\% CI: 1.5-1.8) to 2.2 (95\% CI: 2.0-2.4) in one year follow-up in patients discharged from a hospital in 1999 versus 2006 (Hoang et al. 2011). SMRs were higher for younger schizophrenia patients discharged in 2006 versus the oldest group. For patients below 45 years of age, SMR was 6.2 (95\%, CI 4.9-7.5), and for patients in the group of 65-84 years - 2.0 (95\%CI: 1.7-2.3). In 2006, a higher mortality rate was demonstrated in this age group for men (3.2, 95\%CI: 2.8-3.6) than for women (1.8, 95\%CI: 1.5-2.1). Between 1999 and 2006, the mortality gap increased in the oldest group of schizophrenia patients (65-84 years), while it remained on a stable level in younger patients, versus an age-matched general population. This change resulted from a drop in mortality rates in a general population during the study. A difference in age at death also increased in 19802010, whereas in that period a mean difference in age at death between schizophrenia patients and general population was 11.2 years (95\% CI: 10.9-11.5), and 12.3 years (95\% CI, 11.9-12.7) for men, and 8.1 years (95\% CI, 7.7-8.5) for women. In 1980s it was 7.1 years in total (for men: 8.9 years; for women: 4.1 years) to reach a mean value of 14.7 years in total in the first decade of the 21st century (for men: 15.3 years; for women: 11.4 years) (Nielsen et al. 2010).

A standardised mortality rate for unnatural causes in schizophrenia patients remained at the stable level of 11.6 (9.5-13.7) in 1999 and 11.6 (9.3-13.9) 2006; therefore, MG for these causes of death remained unchanged. On the other hand, MG for natural causes of death increased, and SMR changed from 1.2 (1.1-1.4) to 1.7 (1.5-1.9). This resulted from an increase in mortality from cardiovascular diseases from 1.6 (1.4 to
1.9) to 2.5 (2.1 to 2.9) and respiratory diseases from 3.1 (2.6 to 3.6) to 4.7 (3.8 to 5.6) (Hoang et al. 2011).

\section{DISCUSSION}

A detailed analysis of factors influencing mortality rates in schizophrenia patients is a critical point in assessment of psychiatric care quality. Results of this systematic review of European studies indicate that medical records documenting diagnoses, applied treatment strategies and deaths in patients with schizophrenia are a crucial tool in mortality studies. Medical records availability is reflected in quantity and quality of published studies on mortality in schizophrenia, recently conducted in Northern and Western Europe. Since there are no studies addressing mortality in schizophrenia patients from Middle and Eastern European countries, it is hard to draw conclusions on the influence of changes in psychiatric health care that rely on switching from institutional to community-based care on mortality rates (WHO 2014).

It is surprising to notice that the current studies tend to neglect the influence of the specific risk factors on mortality. WHO considers smoking, obesity, low physical activity, raised glucose levels, high cholesterol level and hypertension as modifiable life-style and healthassociated mortality risk factors (WHO 2009), whereas only few studies present data on the influence of some of the specific factors on mortality in schizophrenia. Moreover, there is a lack of data on the influence of potential interventions to modify these risk factors on mortality. A review of mortality risk factors in firstepisode schizophrenia (Beary et al. 2012) concludes there is a limited amount of data in this field, however it is suggested that interventions targeting such modifiable risk factors as smoking, hypertension and cardiorespiratory fitness could improve the lifespan of persons with schizophrenia. Further studies on modifiable mortality risk factors are encouraged. Kodama et al. (2009) suggest better cardiorespiratory fitness to be associated with lower all-cause mortality and mortality due to coronary heart disease and cardiovascular disease. In recent meta-analysis, Vancampfort et al. (2015) show a significantly reduced cardiorespiratory fitness in persons with schizophrenia, as well as in persons after first psychotic episode, in comparison with general population. Promoting physical activity and improving cardiorespiratory fitness may be a key intervention to reduce the overall and CVD-associated mortality in these patients (Vancampfort et al. 2015). The studies included in our review, however, fail to address this issue: only one study analysed the influence of physical exercise on mortality (Brown et al. 2012), though due to the limitations of data (patients reporting any physical activity may not necessarily do significant amount of exercise), the results were not conclusive enough. Whereas cardiorespiratory fitness as a separate variable has not been considered in the studies comprised in our review. With growing reports on the potential benefits 
of physical activity in these persons, it is therefore necessary to design prospective studies which will include the frames for finding the appropriate behavioural interventions in this area.

Although all analysed studies were of a cohort type, based on data from medical records, they were conducted with different methods, so the comparison of individual study results is largely limited. Differences can be already noticed with respect to the selection of particular patients' populations. Cohorts where patients hospitalised for the first time (usually young) predominate are characterised by higher suicide rate, while in older groups cancer occurrence is higher, and in the oldest groups deaths of cardiovascular diseases predominate (Bushe et al. 2010). On the other hand, improvement in quality of studies on mortality from external causes, particularly in countries without an institution of coroner, can be achieved by thorough analysis of patient records and event circumstances, including information from witnesses and deceased family. In studies included in this review, mortality was usually one of many analysed issues. Therefore, a question arises whether a uniform methodology should be established, so studies conducted in different European countries would be comparable resulting in more generalized conclusions.

Our review confirms the opinion from the World Health Organization Action Plan (WHO 2014) that modern data collection systems are available only in countries with high gross domestic product per capita. The British National Health Service system collecting data on each patient admitted to a hospital since 1980s is an exemplary system for collection of medical data. Due to posing a risk or privacy while gathering medical data, a number of security solutions have been used to protect sensitive data (Lewis 2014, Ward 2014), with also OECD providing their guidelines (OECD 2013).

In general, mortality in schizophrenia is increased versus a reference population, where increase in mortality from individual causes of death is unambiguous (Allebeck 1989, Mortensen \& Juel 1993, Brown 1997, Saha et al. 2007, Tran et al. 2009). The most common causes of death in schizophrenia patients in Europe do not differ significantly from those in the general population, currently being cardiovascular diseases and cancers (Brown et al. 2010, Crump et al. 2013). This conforms to results (Dean \& Thuras 2009) showing cardiovascular diseases as a leading cause, followed by cancers on the second and suicides on the third position, representing: $24 \%, 21 \%$ and $7 \%$ of all deaths, respectively. In 2012 in the general population cardiovascular diseases, cancers, and accidents and poisonings caused nearly 50\%, 20\% and 9\% of all deaths, respectively (WHO 2015).

Deaths for external reasons, with a particular emphasis on suicides, are an important problem in mortality studies, where precise estimation of actual cause of death is low, making evaluation of a suicide rate difficult. This is particularly important for schizophrenia patients where suicides are one of the most common causes of death, with significantly high mortality ratios among all death causes (Brown 1997). In Finland it was noted that a decrease in mortality from suicide may be related to deinstitutionalisation, although results of other studies on the effect of a psychiatric care system on mortality, particularly of unnatural causes, were ambiguous (Rantanen et al. 2009).

Stigmatization of schizophrenia, present also among health care personnel, may limit the patients' access to health care. This is confirmed by a disproportion in mortality from cardiac infarction or cancer in schizophrenia patients and in people without such diagnosis, unfavourable for the former, despite similar prevalence of these disorders (Kisely et al. 2008, Hodgson et al. 2010, Crump et al. 2013). Furthermore, in people who died due to above causes, these disorders were significantly less often diagnosed in schizophrenia patients during their life, and this may indicate lower availability of screening tests and modern diagnostic methods. Also a rate of invasive cardiac procedures in people previously hospitalised for SMI (including schizophrenia) was nearly two times lower versus people without such history (Laursen et al. 2009). Recently, increased mortality rates from cancers have been observed in patients with schizophrenia (Saha et al. 2007, Tran et al. 2009), and this may indicate a delay in diagnostic and therapeutic procedures conducted by other specialists.

Diabetes is the most common chronic comorbidity in schizophrenia, found in $12.5 \%$ of women and $11 \%$ of men during a 7-year follow-up (Crump et al. 2013). Results of British studies indicate an increased risk of death in these patients, with significantly higher morbidity in women. Further studies are necessary to assess this comorbidity, particularly as new rules for contracting services in UK from 2004 significantly improved diabetes detection in schizophrenia patients (Vinogradova et al. 2010). Identifying cardio-metabolic risk factors in each case and regular monitoring of metabolic parameters in patients with schizophrenia, especially when using medications associated with adverse metabolic effects, is recommended (Newcomer 2008).

Results of the study by Tenback et al. (2012), suggesting that FGA cause higher morbidity versus SGA, are confirmed in other contemporary studies (Osborn et al. 2007, Dean \& Thuras 2009).

The quoted studies may indicate a negative mortality tendency (Nielsen et al. 2010, Høye et al. 2011), but this is not confirmed in data from 1990s indicating that the mortality gap between schizophrenia patients and the general population reached a plateau and may decrease (Tiihonen et al. 2009). According to some studies (Laursen 2011, Laursen et al. 2013, Crump et al. 2013), also reduction in life expectancy in schizophrenia was significantly higher than British data, of 14.6 years for men and 9.8 years for women (Chang et al. 2011).

Nevertheless reduction in a mortality gap between the general population and schizophrenia patients should be an objective pursued through improvement in availability of modern health care and actions preven- 
ting suicides and accidents. It seems that in current situation even stopping of adverse tendencies may be perceived as a success. A change in this situation poses a significant challenge to psychiatric health care, but without relevant actions at institutions responsible for the health care system organisation, efforts of specialists alone may be insufficient. Simultaneously, international medical organisations should strive to provide a uniform set of methods for studies on mortality, to facilitate comparisons of study results from different European countries, to develop a joint strategy to reverse unfavourable tendencies for mortality in schizophrenia patients.

\section{CONCLUSIONS}

Published studies on mortality in schizophrenia from 2009-2014 from Northern and Western Europe indicate that SMRs in schizophrenia patients are higher versus the general population, both for natural and for external causes of death. Natural causes, particularly cardiovascular diseases and cancers, prevail over external ones. These rates remain at the stable level or increased slightly, and this may indicate that schizophrenia patients do not benefit from progress in medicine to the same extent as people not suffering from this disease. Diverse study methods cause problems with comparing results from different countries and with drawing precise conclusions.

\section{Acknowledgements:}

The authors would like to thank Dr. Sukanta Saha from the University of Queensland for his invaluable help and advice in preparing the article.

\section{Conflict of interest: None to declare.}

\section{Contribution of individual authors:}

Patryk Piotrowski: design of the study, interpretation of data;

Tomasz M. Gondek: design of the study, search strategy, collection and interpretation of data;

Anna Królicka-Deręgowska: search strategy, collection and assembly of data, design of the tables;

Błażej Misiak: interpretation of results, manuscript editing;

Tomasz Adamowski: manuscript editing;

Andrzej Kiejna: design of the study, interpretation of data.

\section{References}

1. Allebeck P: Schizophrenia: a life-shortening disease. Schizophr Bull 1989; 15:81-9.

2. Auquier P, Lançon C, Rouillon F \& Lader M: Mortality in schizophrenia. Pharmacoepidemiol Drug Saf 2007; 16:1308-12.
3. Beary M, Hodgson R \& Wildgust HJ: A critical review of major mortality risk factors for all-cause mortality in firstepisode schizophrenia: clinical and research implications. J Psychopharmacol 2012; 26(5 Suppl):52-61.

4. Brown S: Excess mortality of schizophrenia. A metaanalysis. Br J Psychiatry 1997; 171:502-8.

5. Brown S, Kim M, Mitchell C\& Inskip H: Twenty-five year mortality of a community cohort with schizophrenia. $\mathrm{Br} J$ Psychiatry 2010; 196:116-21.

6. Brown $S \&$ Mitchell $C$ : Predictors of death from natural causes in schizophrenia: 10-year follow-up of a community cohort. Soc Psychiatry Psychiatr Epidemiol 2012; 47:843-7.

7. Bushe CJ, Taylor M \& Haukka J: Mortality in schizophrenia: a measurable clinical endpoint. J Psychopharmacol 2010; 24(4 Suppl):17-25.

8. Castagnini A, Foldager L \& Bertelsen A: Excess mortality of acute and transient psychotic disorders: comparison with bipolar affective disorder and schizophrenia. Acta Psychiatr Scand 2013; 128:370-5.

9. Chang CK, Hayes RD, Broadbent M, Fernandes AC, Lee $W$, Hotopf $M$ et al.: All-cause mortality among people with serious mental illness (SMI), substance use disorders, and depressive disorders in southeast London: a cohort study. BMC Psychiatry 2010; 10:77.

10. Chang CK, Hayes RD, Perera G, Broadbent MT, Fernandes AC, Lee WE et al.: Life expectancy at birth for people with serious mental illness and other major disorders from a secondary mental health care case register in London. PLoS One 2011; 6:e19590.

11. Crump C, Winkleby MA, Sundquist $K$ \& Sundquist J: Comorbidities and mortality in persons with schizophrenia: a Swedish national cohort study. Am J Psychiatry 2013; 170:324-33.

12. Dean CE \& Thuras PD: Mortality and tardive dyskinesia: long-term study using the US National Death Index. Br J Psychiatry 2009; 194:360-4.

13. De Hert M, Correll CU \& Cohen D: Do antipsychotic medications reduce or increase mortality in schizophrenia? A critical appraisal of the FIN-11 study. Schizophr Res 2010; 117:68-74.

14. Farr W: A report on the mortality of lunatics. J Stat Soc 1841; 4:17-33.

15. Fleischhacker WW, Kane JM, Geier J, Karayal O, Kolluri S, Eng SM et al.: Completed and attempted suicides among 18,154 subjects with schizophrenia included in a large simple trial. J Clin Psychiatry 2014; 75:e184-90.

16. Hjortso S, Butler B, Clemmesen L, Jepsen PW, Kastrup M, Vilmar $T$ et al.: The use of case vignettes in studies of interrater reliability of psychiatric target syndromes and diagnoses. A comparison of ICD-8, ICD-10 and DSM-III. Acta Psychiatr Scand 1989; 80:632-8.

17. Hoang U, Stewart $R \&$ Goldacre MJ: Mortality after hospital discharge for people with schizophrenia or bipolar disorder: retrospective study of linked English hospital episode statistics, 1999-2006. BMJ 2011; 343: $d 5422$.

18. Hoang U, Goldacre MJ \& Stewart R: Avoidable mortality in people with schizophrenia or bipolar disorder in England. Acta Psychiatr Scand 2013; 127:195-201.

19. Hodgson R, Wildgust HJ \& Bushe CJ: Cancer and schizophrenia: is there a paradox? J Psychopharmacol 2010; 24(4 Suppl):51-60. 
20. Høye A, Jacobsen BK \& Hansen V: Increasing mortality in schizophrenia: are women at particular risk? A followup of 1111 patients admitted during 1980-2006 in Northern Norway. Schizophr Res 2011; 132: 228-32.

21. Kisely S, Sadek J, MacKenzie A, Lawrence D \& Campbell LA: Excess cancer mortality in psychiatric patients. Can $J$ Psychiatry 2008; 53:753-61.

22. Kodama S, Saito K, Tanaka S, Maki M, Yachi Y, Asumi M et al.: Cardiorespiratory fitness as a quantitative predictor of all-cause mortality and cardiovascular events in healthy men and women: a meta-analysis. JAMA 2009; 301:2024-35

23. Korkeila J, Lehtinen V, Bijl R, Dalgard O-S, Kovess V, Morgan A et al.: Establishing a set of mental health indicators for Europe. Scand J Public Health 2003; 31:451.

24. Laursen TM, Munk-Olsen T, Agerbo E, Gasse C \& Mortensen PB: Somatic hospital contacts, invasive cardiac procedures, and mortality from heart disease in patients with severe mental disorder. Arch Gen Psychiatry 2009; 66:713-20.

25. Laursen TM: Life expectancy among persons with schizophrenia or bipolar affective disorder. Schizophr Res 2011; 131:101-4

26. Laursen TM, Munk-Olsen T \& Gasse C: Chronic somatic comorbidity and excess mortality due to natural causes in persons with schizophrenia or bipolaraffective disorder. PLoS One 2011; 6:e24597.

27. Laursen TM, Wahlbeck K, Hällgren J, Westman J, Ösby $U$, Alinaghizadeh $H$ et al.: Life expectancy and death by diseases of the circulatory system in patients with bipolar disorder or schizophrenia in the Nordic countries. PLoS One 2013; 8:e67133.

28. Laursen TM, Mortensen PB, MacCabe JH, Cohen D \& Gasse C: Cardiovascular drug use and mortality in patients with schizophrenia or bipolar disorder: a Danish population-based study. Psychol Med 2014; 44:1625-37.

29. Lewis G: Better information means better care, accessed 2 May 2014: http://www.england.nhs.uk/2014/01/15 /geraint-lewis/

30. Mittoux A, Tanghøj $P \&$ Moore N: Exploring the potential prognostic effect of various country-specific health service data on all-cause mortality using data from a large prospective study in schizophrenia. Pharmacoepidemiol Drug Saf 2013; 22:925-32.

31. Mortensen PB \& Juel K: Mortality and causes of death in first admitted schizophrenic patients. Br J Psychiatry 1993; 163:183-9.

32. Newcomer JW: Metabolic syndrome and mental illness. Am J Manag Care 2007; 13(7 Suppl): S170-7. Erratum in: Am J Manag Care 2008; 14:76.

33. Nielsen RE, Uggerby AS, Jensen SO \& McGrath JJ: Increasing mortality gap for patients diagnosed with schizophrenia over the last three decades--a Danish nationwide study from 1980 to 2010. Schizophr Res 2013, 146:22-7.

34. OECD: Strengthening Health Information Infrastructure for Health Care Quality Governance: Good Practices, New Opportunities and Data Privacy Protection Challenges, OECD Health Policy Studies, OECD Publishing, Paris. Accessed 17 May 2014:http://www.keepeek.com/DigitalAsset-Management/oecd/social-issues-migrationhealth/strengthening-health-information-infrastructurefor-health-care-quality-governance_9789264193505en\#page2
35. Osborn DP, Levy G, Nazareth I, Petersen I, Islam A \& King MB: Relative risk of cardiovascular and cancer mortality in people with severe mental illness from the United Kingdom's General Practice Research Database. Arch Gen Psychiatry 2007; 64:242-9.

36. Prince M, Patel V, Saxena S, Maj M, Maselko J, Phillips MR et al.: No health without mental health. Lancet 2007; 370:859-77.

37. Rantanen H, Koivisto AM, Salokangas RK, Helminen M, Oja H, Pirkola $S$ et al.: Five-year mortality of Finnish schizophrenia patients in the era of deinstitutionalization. Soc Psychiatry Psychiatr Epidemiol 2009; 44:135-42.

38. Saha S, Chant D \& McGrath J: A systematic review of mortality in schizophrenia: is the differential mortality gap worsening over time? Arch Gen Psychiatry 2007; 64:1123-31.

39. Samele C, Frew $S$ \& Urquia N: Mental Health Systems in the European Union Member States,Sstatus of Mental Health in Populations and Benefits to be Expected from Investments Into Mental Health: European Profile of Prevention and Promotion of Mental Health (EuroPoPPMH). European Commision, Executive Agency for Health and Consumers Tender, 2013.

40. Schmidt LM, Hesse $M \&$ Lykke J: The impact of substance use disorders on the course of schizophrenia-a 15-year follow-up study: dual diagnosis over 15 years. Schizophr Res 2011; 130:228-33.

41. Schoepf D, Potluri R, Uppal H, Natalwala A, Narendran P \& Heun R: Type-2 diabetes mellitus in schizophrenia: increased prevalence and major risk factor of excess mortality in a naturalistic 7-year follow-up. Eur Psychiatry 2012; 27:33-42.

42. Suvisaari J, Partti K, Perälä J, Viertiö S, Saarni SE, Lönnqvist $J$ et al.: Mortality and its determinants in people with psychotic disorder. Psychosom Med 2013; 75:60-7.

43. Sykes FRS: Statistics of the metropolitan commission in lunacy. J Stat Soc 1840; 3:22-36.

44. Tabita B, de Santi $M G$ \& Kjellin L: Criminal recidivism and mortality among patients discharged from a forensic medium secure hospital. Nord J Psychiatry 2012; 66:283-9.

45. Talaslahti T, Alanen HM, Hakko H, Isohanni M, Häkkinen $U$ \& Leinonen E: Mortality and causes of death in older patients with schizophrenia. Int $J$ Geriatr Psychiatry 2012; 27:1131-7.

46. Tenback D, Pijl B, Smeets H, Os Jv \& Harten Pv: Allcause mortality and medication risk factors in schizophrenia: a prospective cohort study. J Clin Psychopharmacol 2012; 32:31-5.

47. Tiihonen J, Lönnqvist J, Wahlbeck K, Klaukka T, Niskanen L, Tanskanen A et al.: 11-year follow-up of mortality in patients with schizophrenia: a population-based cohort study (FIN11 study). Lancet 2009; 374:620-7.

48. Tiihonen J, Suokas JT, Suvisaari JM, Haukka J, Korhonen P: Polypharmacy with antipsychotics, antidepressants, or benzodiazepines and mortality in schizophrenia. Arch Gen Psychiatry 2012; 69:476-83.

49. Tran E, Rouillon F, Loze JY, Casadebaig F, Philippe A, Vitry $F$ et al.: Cancer mortality in patients with schizophrenia: an 11-year prospective cohort study. Cancer 2009; 115:3555-62.

50. Vancampfort D, Rosenbaum S, Probst M, Soundy A, Mitchell AJ, De Hert $M$ et al.: Promotion of cardiorespiratory fitness in schizophrenia: a clinical overview and meta-analysis. Acta Psychiatr Scand 2015; 132:131-43. 
51. Vinogradova Y, Coupland C, Hippisley-Cox J, Whyte S \& Penny C: Effects of severe mental illness on survival of people with diabetes. Br J Psychiatry 2010; 197:272-7.

52. Ward A: Privacy fears over NHS database in aid of medical research. Accessed 11 February 2014: http://www.ft.com/intl/cms/s/0/bc3a9962-8944-11e3-bb5f00144feab7de.html

53. WHO: Leading causes of death in Europe: fact sheet, accessed 9 August 2015: http://www.euro.who.int/_data/ assets/pdf_file/0004/185215/Leading-causes-of-death-inEurope-Fact-Sheet.pdf

54. WHO: Global health risks: mortality and burden of disease attributable to selected major risks, Geneva,
World Health Organization. Accessed 30 October 2016: http://www.who.int/healthinfo/global_burden_disease/Glo balHealthRisks_report_full.pdf

55. WHO: Mental health action plan 2013-2020, accessed 19 July 2014, http://apps.who.int/iris/bitstream/10665/89966/1/ 9789241506021_eng.pdf.

56. Yasamy MT, Cross A, McDaniell E \& Saxena S: Living with Schizophrenia. Section V: Time to act. Living a healthy life with schizophrenia: paving the road to recovery. World Health Organization. Accessed: 30 October 2016: http://www.who.int/mental_health/worldmental-health-day/paper_wfmh.pdf
Correspondence:

Tomasz M. Gondek, MD

Department of Psychiatry, Wroclaw Medical University 10 Pasteur Street, 50-367 Wroclaw, Poland

E-mail: gondektm@gmail.com 